\title{
FUSÃO ENTRE TÉCNICAS DE EXTRAÇÃO DE ATRIBUTOS NA CLASSIFICAÇÃO DE ISOLADORES DE REDES DE DISTRIBUIÇÃO USANDO REDES NEURAIS
}

\author{
André P. Marotta* Eduardo F. de Simas Filho*, Ricardo M. Prates ${ }^{\dagger}$, Rodrigo P. Ramos ${ }^{\dagger}$ \\ * Laboratório de Sistemas Digitais, PPGEE, UFBA \\ Salvador, Bahia, Brasil \\ ${ }^{\dagger}$ Departamento de Engenharia Elétrica, UNIVASF \\ Juazeiro, Bahia, Brasil
}

Emails: andre.marotta@ufba.br, eduardo.simas@ufba.br, ricardo.prates@univasf.br, rodrigo.ramos@univasf.br

\begin{abstract}
The present work provides a methodology for automated image processing and classification of four types of insulators used in Overhead Power Distribution Lines (OPDLs). To this end, a didactic distribution network was developed to capture photos of the insulators at an external environment as well as a studio to acquire images at a controlled environment. Subsequently, the image attributes where extracted and used as inputs for diferent trained classifiers such as neural networks, suport vector machine, decision tree, n-nearest neigbors, naive-bayes and hidden markov model, for a gradual mixing ofthe databases (studio and external imagens). The classification efficiencies where compared considering the application of principal components analysis (PCA) to identify the minimum information required for the classification.
\end{abstract}

Keywords - Overhead Power Distribution Lines (OPDLs), Insulators, Digital Image Processing, Artificial Neural Networks, PCA.

Resumo - O presente trabalho apresenta uma metodologia para processamento automatizado de imagens e classificação de quatro tipos de isoladores utilizados em redes de distribuição aéreas de média tensão (RDAs). Para isso, foi desenvolvida uma rede de distribuição didática para coletar fotos dos isoladores em um ambiente externo e também um estúdio para aquisição de imagens em ambiente controlado. Posteriormente, os atributos de imagem foram extraídos e utilizados como entrada para diferentes classificadores treinados, como redes neurais, máquina de vetor de suporte, árvore de decisão, n-vizinhos próximos, naive bayes e modelo oculto de Markov, para uma mistura gradual entre as bases de dados (estúdio e imagens externas). As eficiências de classificação foram comparadas considerando a aplicação da análise de componentes principais (ACP) para identificar as informações mínimas necessárias para a classificação.

Palavras-chave - Redes de Distribuição Aéreas (RDAs), Isoladores, Processamento Digital de Imagem (PDI), Redes Neurais Artificiais (RNA), Análise de Componentes Principais (ACP).

\section{Introdução}

A robótica de inspeção industrial é um campo relativamente novo, porém, promissora área da engenharia industrial (Electric, 2018). Os recentes avanços na área de inteligência artificial embarcada (Kuzum et al., 2011), a crescente disponibilidade de processamento inteligente tanto em software (Fulcher, 2008) quanto em hardware (Desoli et al., 2016) e a busca de eficiência operacional em sistemas de engenharia fazem com que a robótica de inspeção seja cada dia mais popular.

Nas redes de distribuição Aéreas (RDAs) de energia elétrica em média tensão, as atividades de inspeção corretiva e preventiva muitas vezes são realizadas na modalidade de inspeção visual, "por patrulha a pé", ou assistidas por helicóptero (Pereira, 2009). Essas atividades são normalmente baseadas em métodos empíricos de avaliação, podendo resultar em falhas de cadastro e planejamento inadequado das ações de manutenção. A consequência direta destas inconsistências é o aumento nos custos com logística e manutenção. A depender do caso, um erro na avaliação de isoladores pode interferir na qualidade de fornecimento de energia para o consumidor final.
Existem na literatura trabalhos como (Sampedro et al., 2014), (Souza, 2016), (Ling, 2018) que têm por finalidade desenvolver tecnologias de inspeção com inteligência embarcada em robôs móveis aéreos (drones) para redes de distribuição. Uma ilustração do uso de drone para inspeção em rede de distribuição é mostrada na Figura 1.

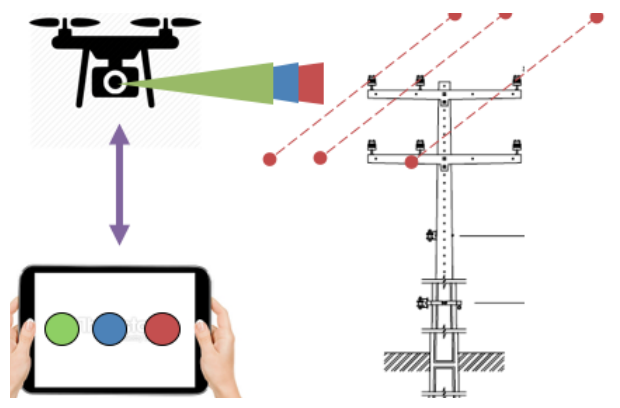

Figura 1: Ilustração da utilização de um drone para inspeção em uma rede elétrica de distribuição.

Neste trabalho, são utilizadas técnicas de Processamento Digital de imagen (PDI) e de inteligência computacional com o intuito de classificar 
diferentes tipos de isoladores elétricos de média tensão.

No trabalho (Prates et al., 2017) foi realizado um estudo inicial de classificação utilizando uma rede neural para 3 tipos de isoladores utilizando um único método de extração de atributos. Para realizar o estudo descrito em (Marotta et al., 2018) foi projetado e desenvolvido um estúdio fotográfico. Neste caso, o intuito foi diversificar os métodos de extração de atributos e verificar a influência da inclinação das imagens obtidas do estúdio na classificação. Foi considerada também a variação da quantidade de atributos por meio da compactação por análise de componentes principais.

O objetivo principal desta pesquisa foi comparar o funcionamento das técnicas de extração de atributos implementadas em (Marotta et al., 2018) quando aplicadas em imagens obtidas tanto em um ambiente controlado quanto em um ambiente externo, a fim de estudar a viabilidade de utilizar imagens do ambiente controlado para classificar isoladores em um ambiente externo.

\section{Material e Métodos}

\subsection{Material}

As amostras de teste utilizadas nos experimentos consistem em 4 tipos de isoladores de redes de distribuição que operam na faixa de $15 \mathrm{kV}$, comumente utilizados em instalações rurais e urbanas. Os tipos de isoladores são: de porcelana (IPN), saia baiana (ISB), isolador de vidro (IVD) e o polimérico (IPL). Imagens de amostras dos isoladores utilizados são exibidas na Figura 2. (a)

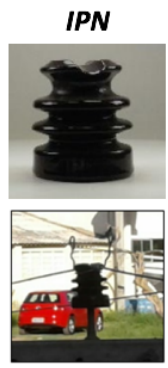

ISB
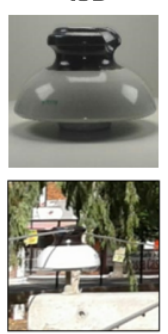

IVD
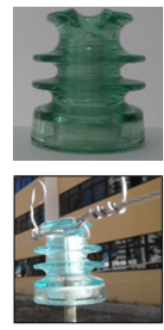

IPL
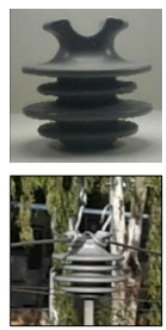

Figura 2: Amostras de imagens de isoladores de: porcelana (IPN), saia baiana (ISB), polimérico (IPL), pino de vidro (IVD) obtidas em (a) Ambiente Controlado e (b) Ambiente Externo.

As imagens adquiridas dos isoladores no ambiente controlado formam a base de dados $\mathrm{C} 1$ e as imagens dos isoladores no ambiente externo formam a base de dados $\mathrm{C} 2$.

\subsection{Métodos}

A metodologia para classificação do tipo de isolador (IPN, ISB, IPL, ou IVD) desenvolvida neste trabalho pode ser dividida em 4 partes:
1. Projeto e construção de sistemas para aquisição de imagens.

2. Extração de características e fusão dos atributos implementados.

3. Treino/Teste de classificadores com a base de dados C1.

4. Teste dos classificadores treinados na etapa anterior com os dados da base de dados C2.

As referidas etapas serão listadas nas seções 3 e 4 .

\section{Metodologia para aquisição de imagens}

\subsection{Ambiente Controlado}

A fim de atender aos objetivos da pesquisa, foi desenvolvido um estúdio para aquisição de imagens em um ambiente controlado, chamado de SEAI (Sistema Experimental de Aquisição de Imagens), apresentado na Figura 3.
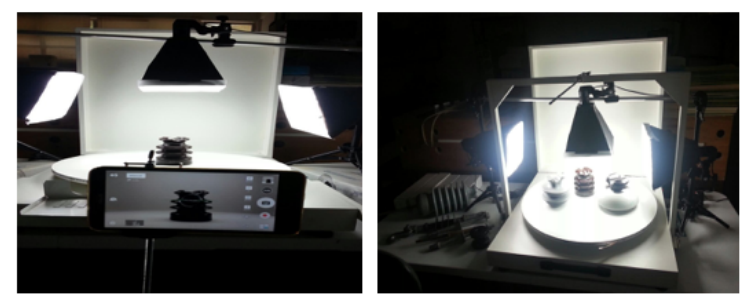

Figura 3: Estúdio SEAI (Sistema Experimental de Aquisição de imagens).

Este estúdio foi montado em MDF sendo composto por uma base quadrada dobrável com lateral de $700 \mathrm{~mm}$ e pés de apoio. Foi instalado um disco de giro livre com diâmetro de $610 \mathrm{~mm}$ para permitir a aquisição de imagens de um objeto rotacionando-o entre 0 e 360 graus em torno do seu próprio eixo $(\phi)$. Na parte frontal, foi instalado um suporte para fixação ajustável em altura de um Smart Phone para que fosse possível obter fotos em ângulos de 0 a 90 graus em relação à vista frontal do objeto $(\theta)$. Uma ilustração do SEAI é mostrada na Figura 4.

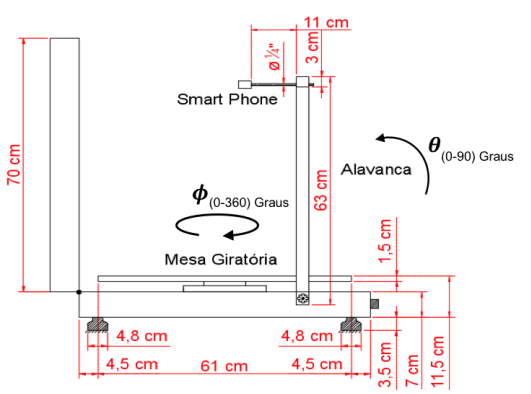

Figura 4: SEAI: Mesa Giratória e Suporte do Smart Phone. 
Adicionalmente, foi instalado um sistema de iluminação com 3 lâmpadas de luz corrigida utilizadas em estúdios de fotografia, fixas em difusores, conforme ilustrado na Figura 5.

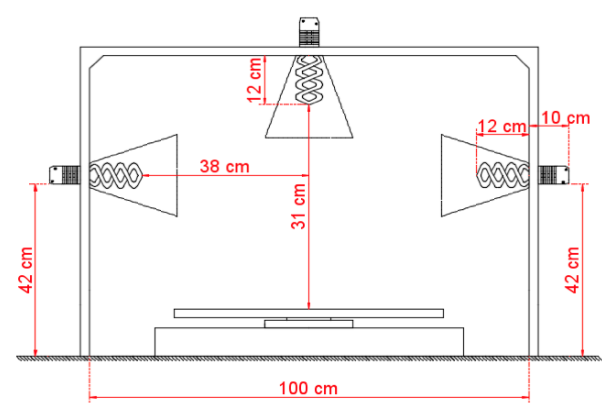

Figura 5: SEAI: Sistema de iluminação controlada.

\subsection{Ambiente Externo}

A fim de reproduzir uma rede de distribuição realista em um ambiente externo, foi desenvolvida uma rede chamada de RDD (Rede de Distribuição Didática). O sistema consiste de 3 postes com altura de 1,5 metros para facilitar a instalação dos isoladores e acessórios de fixação, bem como a aquisição das imagens.

Os postes foram instalados a uma distância de 15 metros entre si. O sistema de ancoragem foi configurado supondo a instalação de 3 isoladores por poste. Assim, foram montados 3 cabos de força para proporcionar a passagem de um circuito trifásico e os isoladores foram fixados ao cabo através de um aramado, conforme detalhado na Figura 6.

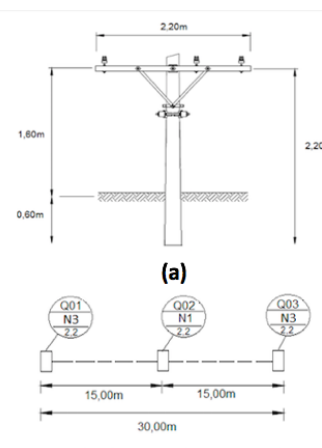

(b)

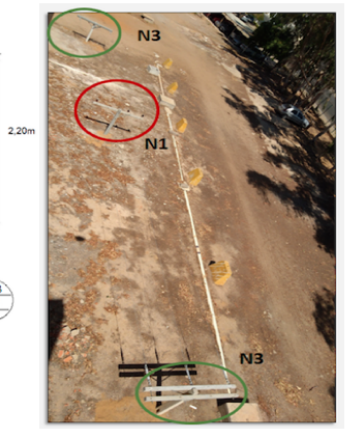

(c)
Figura 6: Rede RDD (Rede de Distribuição Didática) (a) Diagrama da vista Frontal (b) Diagrama da vista Longitudinal (c) Instalação da RDD.

\subsection{Construção da Base de Dados}

Para a aquisição das imagens foi utilizado um Smart Phone com câmera de 13 MP, 256 PPI e formato 16:9. Para a construção da base de dados e para cada isolador, em relação a $\phi$ (para um $\theta$ fixo), foram adquiridas 194 imagens (espaçamento de 1,67 graus). Em relação a $\theta$ foram adquiridas imagens a cada 10 graus (0 a 90 graus) repetindo o procedimento $\phi$ totalizando 1940 imagens por isolador. Por fim, as imagens foram recortadas manualmente e redimensionadas para o formato 1:1 e dimensões 1500 x 1500 pixels.

Para a construção da base de dados C1 correspondente ao ambiente controlado, foram selecionados 4 tipos de isoladores. Assim, das imagens adquiridas através do estúdio, foram selecionadas 40 imagens a cada variação de $\theta$, totalizando 400 amostras para cada classe. Para a construção de C2 foram instalados os mesmos 4 tipos de isoladores na RDD e adquiridas 400 imagens de cada um deles para diferentes valores de $\phi$ e $\theta$. Por fim, todas as imagens foram redimensionadas para o formato 1:1 e dimensões $224 \times 224$ pixels.

\section{Extração de Atributos e Classificação de Imagens}

\subsection{Método da Fusão entre Atributos (FUS)}

O conjunto de técnicas de extração de atributos utilizado no trabalho, consiste na fusão das técncas Geométrica (GEO), Estatística (EST) e Domínio da freqência (FRQ) (Marotta et al., 2018), (Gonzalez and Woods, 2012), (Backes et al., 2016), denominada Fusão (FUS).

A técnica GEO consitste da extração de características geométricas do objeto de interesse na imagem, formando 5 atributos (G1, G2, G3, G4 e G5). A técnica EST consiste em obter os histogramas da imagem no padrão RGB (HR, HG, HB), na escala de cinza (HS) e no padrão YCbCr $(\mathrm{HY}$, $\mathrm{HCr}, \mathrm{HCb}$ ) calculando respectivamente os momentos estatísticos média (ME), variância (VA), curtose (CR) e assimetria (AS), bem como a entropia (ET) e energia (EN) de cada um deles, formando o conjunto de atributos. Já a técnica FRQ consiste da extração da assinatura de contorno e desta, extrair atributos com menores dimensões, utilizando as transformadas wavelet (daubechies db2) (DW1) e de fourier (DF) do sinal, tanto no domínio XY quando nos contornos parciais em $\mathrm{X}$ e em Y, calculando a transformada wavelet, e respectivas entropia e energia de cada um dos contornos parciais formando os vetores (DW2X, DW2Y) obtendo assim um conjunto de atributos de contorno. O resumo do conjunto de atributos extraídos em cada uma das técnicas descritas está ilustrado no diagrama da Figura 7.

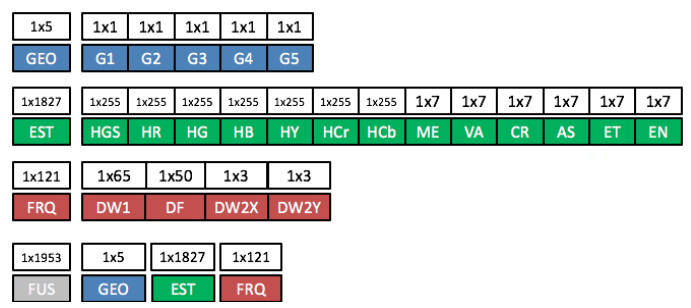

Figura 7: Diagrama que resume os atribudos extraídos através dos métodos GEO, EST, FRQ e FUS. 
A implementação da técnica FUS teve como objetivo identificar se o aumento de dimensionalidade pode promover uma menor dispersão de resultados e uma melhoria na qualidade da informação extraída das amostras de imagens. Neste sentido, foi posteriormente realizada uma compressão de dados através da Análise de Componentes Principais (PCA) (Papoulis and Pillai, 2002), uma técnica estatística que tem como objetivo a busca de uma transformação linear, na qual a maior parte da energia esteja concentrada em um número reduzido de componentes.

\subsection{Classificadores}

A utilização de imagens em um ambiente controlado para classificar objetos em um ambiente real permite o desenvolvimento de um sistema de classificação com menor custo e menor tempo, se comparado a um sistema baseado somente em aquisição de imagens no campo. Este tipo de controle permite uma compreensão de como se comporta o conjunto de atributos extraídos das imagens dos isoladores, sem a interferência de objetos externos que produzem uma quantidade de dados que não são do objeto e portanto não são de interesse do aprendizado da máquina. O estudo comparativo entre a eficiência da classificação em um ambiente controlado e um ambiente externo requer considerar que ocorre um aumento de dificuldade no processo de extração de atributos, na medida em que o objeto de interesse se encontra em um cenário mais complexo.

Com o intuto de mitigar este problema, foi realizado neste trabalho um processo de mistura gradual de dados entre $\mathrm{C} 1$ e C2. Para tal, foram projetados 4 classificadores neurais, utilizando o método FUS. O primeiro foi treinado usando atributos extraídos de $\mathrm{C} 1$, e ao restante foi adicionado ao conjunto de treino/teste $10 \%, 20 \%$ e $30 \%$ dos primeiros dados de C2. Na etapa de teste 1 , os 4 conjuntos de classificadores foram testados com as imagens de teste de $\mathrm{C} 1$, obtendo a saída 1 denominada FUS-C1. Na etapa de teste 2, estes classificadores que foram treinados com os dados de $\mathrm{C} 1$, foram testados com as imagens de $\mathrm{C} 2$, obtendo a saída 2 denominada FUS-C2. O procedimento de mistura de dados e teste está apresentado na Figura 8.

O principal classificador utilizado foi a Rede Neural (RNA-MLP) tipo Perceptron multicamadas (MLP - Multilayer Perceptron), com única camada oculta, treinamento pelo método da retro-propagação de erro e função de ativação Sigmoidal. Também foram utilizados a Máquina de Vetor de Suporte (SVM-ECOC), Árvore de Decisão (DTREE), Floresta Aleatória (R-FOREST), Modelo Oculto de Markov (HMM), Náive Bayes (BAYES), N-Vizinhos Próximos (KNN) e o 1Vizinho Próximo (1NN) (Faceli et al., 2011). Os classificadores foram treinados utilizando o mé-

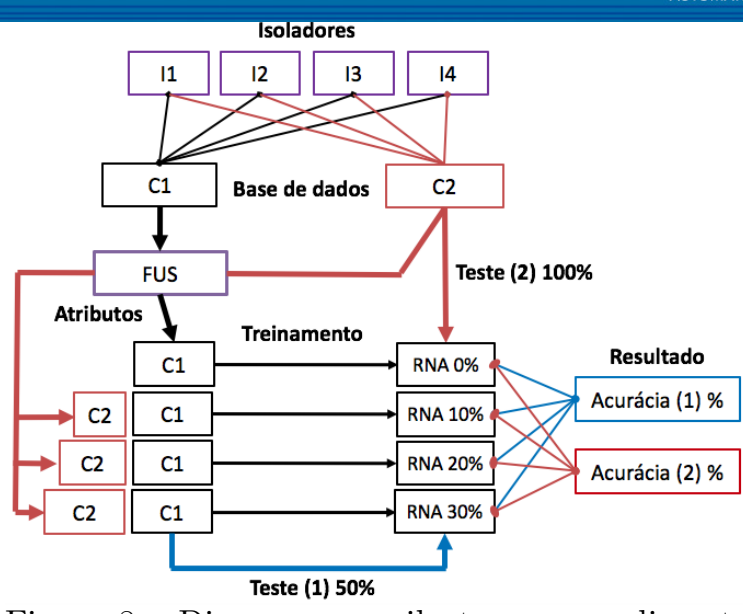

Figura 8: Diagrama que ilustra o procedimento de mistura de dados adotado para o treino e teste das Redes Neurais.

todo de validação cruzada $K$-fold Leave-M-out com $50 \%$ das amostras para teste e $50 \%$ do conjunto para treino de 100 classificadores, fazendo um novo sorteio a cada 10 treinamentos, variando de 10 a 100 o número de neurônios na camada oculta e índices dos demais classificadores e tendo a acurácia como figura de mérito.

\section{Resultados}

\subsection{Resultados Obtidos}

\subsubsection{Estudo 1 : Fusão entre atributos}

Para a primeira etapa (análise da fusão entre atributos) foram realizados testes das RNAs treinadas com os dados de $\mathrm{C} 1$ para os métodos GEO EST, FRQ e FUS. Os resultados são apresentados na Figura 9.

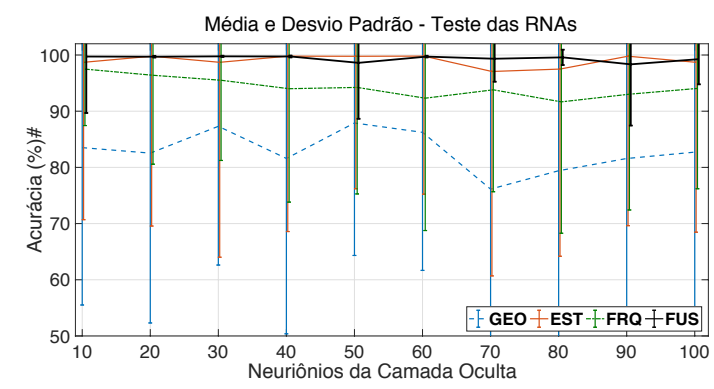

Figura 9: Resultados da avaliação de média e desvio padrão da classificação dos dados de $\mathrm{C} 1$ através dos métodos GEO, EST, FRQ e FUS.

\subsubsection{Estudo 2 : Melhor classificador}

$\mathrm{Na}$ etapa complementar, foi realizado o treino e teste de 8 tipos de classificadores, utilizando os testes (FUS-C1) e (FUS-C2) considerando a mistura gradual dos dados de treinamento entre $\mathrm{C} 1 \mathrm{e}$ C2. Os resultados estão apresentados na Tabela 1. Os resultados indicam que somente os classificadores baseados em RNA apresentaram sensibilidade significativa à mistura dos dados. 
Tabela 1: Resultados de melhor acurácia dos 8 tipos de Classificadores utilizando o método FUS com a mistura de dados de treino entre $\mathrm{C} 1$ e $\mathrm{C} 2$.

\begin{tabular}{|c|c|c|c|c|c|}
\hline Tipo & $\begin{array}{c}\text { Tr C1 } \\
\text { Te C1 }\end{array}$ & $\begin{array}{c}\text { Tr C1 } \\
\text { Te C2 }\end{array}$ & $\begin{array}{c}\text { Tr C1+C2 } \\
\text { Te C2 }\end{array}$ & $\begin{array}{c}\text { Tr C1+C2 } \\
\text { Te C2 } 2\end{array}$ & $\begin{array}{c}\text { Tr C1+C2 } \\
\text { Te C2 }\end{array}$ \\
\hline ANN-MLP & 99,54 & 55.00 & 59.22 & 64.86 & 70.02 \\
\hline SVM-ECOC & 97,36 & 25,10 & 30.07 & 27.11 & 32.05 \\
\hline D-TREE & 65,24 & 33,21 & 39.06 & 44.11 & 45.18 \\
\hline R-FOREST & 67,15 & 49,13 & 30.12 & 29.04 & 28.07 \\
\hline HMM & 60,12 & 42,18 & 40.25 & 41.06 & 39.21 \\
\hline BAYES & 55,34 & 25,11 & 29.33 & 34.81 & 36.07 \\
\hline KNN & 57,08 & 28,19 & 33.02 & 32.10 & 31.06 \\
\hline 1NN & 43,70 & 31,23 & 33.70 & 32.67 & 42.19 \\
\hline
\end{tabular}

\subsubsection{Estudo 3: Mistura de dados sem compressão}

Para a terceira etapa (sem compressão) foram realizados os dois testes das RNAs treinadas com os dados organizados como na Figura 8. Os resultados são apresentados na Figura 10.

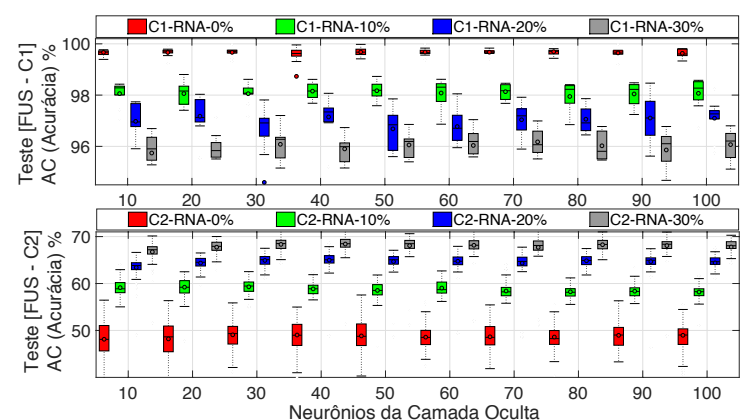

Figura 10: Resultados do Teste (1) (FUS-C1) e do Teste (2) (FUS-C2) das RNAs treinadas.

\subsubsection{Estudo 4: Mistura de dados com compressão}

Para a quarta etapa (com compressão) foram realizados os dois testes das RNAs treinadas com 3 níveis distintos de compressão por PCA (Análise de Componentes Principais). Os níveis de compressão e redução de dimensionalidade estão descritos na Tabela 2.

Tabela 2: Fatores de compressão de sinais FUS pelo método PCA.

\begin{tabular}{|c|c|c|}
\hline ID & $\begin{array}{c}\text { Compressão } \\
\text { PCA }\end{array}$ & Atributos \\
\hline Sem Compressão & $100 \%$ & 1953 \\
\hline$P C A_{(3)}$ & $99,9 \%$ & 912 \\
\hline$P C A_{(4)}$ & $99,4 \%$ & 383 \\
\hline$P C A_{(5)}$ & $99,1 \%$ & 45 \\
\hline
\end{tabular}

Todos os 3 níveis de compressão mantiveram cerca de $99 \%$ de energia do sinal retida após a redução da dimensionalidade, tanto para $\mathrm{C} 1$ quanto para $\mathrm{C} 2$.

Os melhores resultados estão apresentados nas Figuras 11, 12 e 13.

\subsection{Análise dos Resultados Obtidos}

No primeiro estudo (fusão entre atributos) foi verificado que o método FUS foi capaz de reduzir

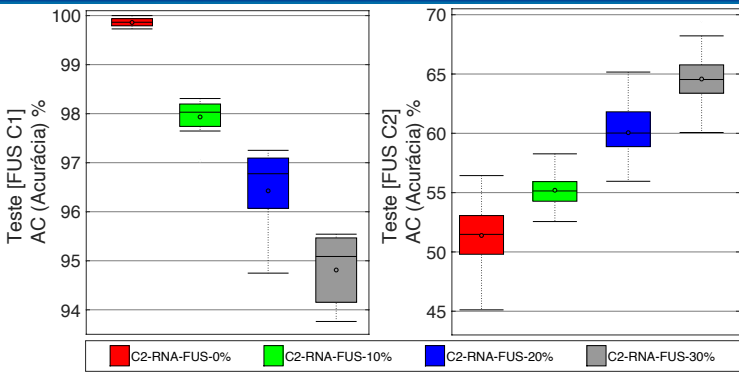

Figura 11: Resultados do Teste (1) (FUS-C1) e do Teste (2) (FUS-C2) das RNAs treinadas com compressão por PCA N3 e 30 neurônios na camada oculta.

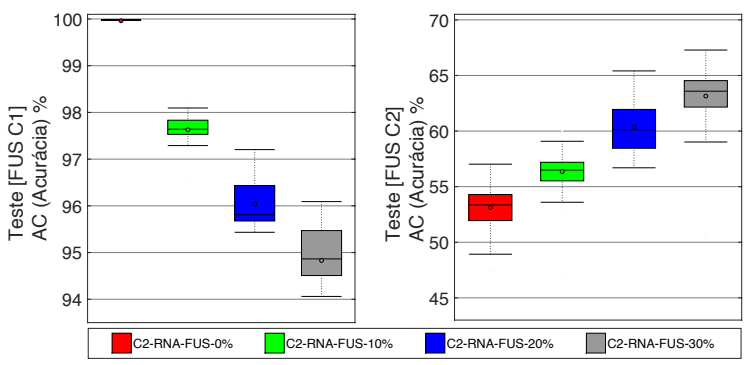

Figura 12: Resultados do Teste (1) (FUS-C1) e do Teste (2) (FUS-C2) das RNAs treinadas com compressão por PCA N4 e 30 neurônios na camada oculta.

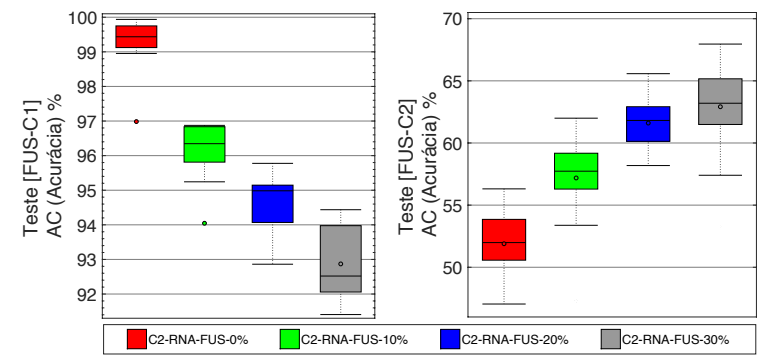

Figura 13: Resultados do Teste (1) (FUS-C1) e do Teste (2) (FUS-C2) das RNAs treinadas com compressão por PCA N5 e 30 neurônios na camada oculta.

o desvio nos resultados de classificação se comparado aos métodos GEO, EST, FRQ. No segundo estudo, a título de comparação, foram treinados 8 tipos de classificadores sem considerar a mistura de dados no treinamento entre $\mathrm{C} 1$ e $\mathrm{C} 2$, onde foi verificado que a RNA-MLP apresentou melhor acurácia de classificação tanto no teste (1) (FUSC1) quanto no teste (2) (FUS-C2). No terceiro estudo (sem compressão) através do método FUS, foi verificado que à medida que ocorre a mistura dos dados de $\mathrm{C} 1$ e $\mathrm{C} 2$, ocorre uma leve piora na acurácia da classificação no teste (1) (FUS-C1). Em compensação, no teste (2) (FUS-C2), a acurácia da classificação tende a variar de $50 \%$ para o caso sem acréscimo de dados de $\mathrm{C} 2$, para cerca de $70 \%$ para o caso com $30 \%$ de acréscimo de dados de C2. No quarto estudo (com compressão) foi verificado que à medida que ocorre a mistura dos dados de $\mathrm{C} 1$ e $\mathrm{C} 2$, ocorre um decréscimo gradual na acurácia de classificação no teste (1) (FUS-C1) decaindo para valores entre $92 \%$ a $95 \%$. Em com- 
pensação, no teste (2) (FUS-C2), a acurácia de classificação manteve a variação de $50 \%$ a $70 \%$ para o caso do acréscimo gradual de dados de $\mathrm{C} 2$. O método de extração de atributos FUS possui uma alta dimensionalidade, porém foi verificado que ele é capaz de reduzir o desvio padrão, garantindo uma baixa dispersão nos resultados de classificação. A posterior compressão por PCA, demonstrou que é possivel reduzir a dimensionalidade do conjunto de atributos, mantendo aproximadamente a mesma faixa de acurácia de classificação.

\section{Conclusões}

Este trabalho teve como proposta o estudo da classificação de isoladores de redes de distribuição em um ambiente externo, com base em imagens adquiridas em um ambiente controlado. Para tal, foram realizados 4 estudos de caso: a fusão entre técnicas de extração de atributos, a comparação entre classificadores, a mistura entre as bases de dados de C1 e C2 e a compressão de dados. A partir dos resultados obtidos, conclui-se que o método FUS aumenta a dimensionalidade dos dados de treino, mas garante menor dispersão dos resultados de classificação, tendo a rede neural obtido as melhores acurárias de classificação em comparação aos demais classificadores. A mistura gradual de dados entre C1 e C2 melhorou a acurária de classificação das imagens do ambiente externo e a compressão de dados nas faixas testadas reduziu a dimensionalidade dos atributos mantendo os mesmos níveis aproximados de acurácia de classificaçã. Para trabalhos futuros pretende-se comparar técnicas de extração de atributos com base em outros métodos como o heurístico e convlucional, bem como estudar o efeito das técnicas de remoção de fundo na extração de atributos das imagens do ambiente externo.

\section{Agradecimentos}

O presente trabalho foi realizado com apoio da Coordenação de Aperfeiçoamento de Pessoal de Nível Superior - Brasil (CAPES) - Código de Financiamento 001.

\section{Referências}

Backes, A. R., Junior, S. and de Mesquita, J. J. (2016). Introdução à Visão Computacional Usando MATLAB, Alta Books Editora.

Desoli, G., Tomaselli, V., Plebani, E., Urlini, G., Pau, D., Dalto, V., Majo, T., De Ambroggi, F., Boesch, T., Singh, S.-p. et al. (2016). The orlando project: A $28 \mathrm{~nm}$ fd-soi low memory embedded neural network asic, International Conference on Advanced Concepts for Intelligent Vision Systems, Springer, pp. 217-227.

Electric, G. (2018). Company will use crawling flying robots ai predictive analytics inspect hard reach-places. Disponível

\begin{abstract}
em: "http://www.ge.com/reports/ new-company-will-use-crawling-/ /flying-robots-ai-predictive-। \analytics-inspect-hard-reach-places.", Acesso em: 2018-05-30.
\end{abstract}

Faceli, K., Lorena, A. C., Gama, J., Carvalho, A. C. P. d. L. et al. (2011). Inteligência artificial: Uma abordagem de aprendizado de máquina.

Fulcher, J. (2008). Computational intelligence: an introduction, Springer, pp. 3-78.

Gonzalez, R. C. and Woods, R. E. (2012). Digital image processing.

Kuzum, D., Jeyasingh, R. G., Lee, B. and Wong, H.-S. P. (2011). Nanoelectronic programmable synapses based on phase change materials for brain-inspired computing, Nano letters 12(5): 2179-2186.

Ling, Zenan, Q. R. C. (2018). Accurate realtime sel-blast glass insulator location method based on faster r-cnn and u-net with aerial images, (CS.CV) Computational Sistems and Computer Vision Journal $\mathbf{I}$.

Marotta, A. P., Simas Filho, E. F. d., Ramos, R. P. and Prates, R. M. (2018). Estudo da influência do Ângulo de inclinação na classificação de isoladores usando redes neurais e processamento de imagem, Conferência $\mathrm{Na}$ cional em Comunicações, Redes e Segurança da Informação VIII: 101-102.

Papoulis, A. and Pillai, S. U. (2002). Probability, random variables, and stochastic processes, Tata McGraw-Hill Education.

Pereira, M. J. (2009). Engenharia de manutenção: teoria e prática, Editora Ciência Moderna.

Prates, R. M., Simas Filho, E. F. d., Cerqueira, J. J. F. and Ramos, R. P. (2017). Desenvolvimento de metodologia inteligente para classificação de tipos de isoladores em redes de distribuição, Simpósio Brasileiro de Telecomunições e Processamento de Sinais XXXV: 398-402.

Sampedro, C., Martinez, C., Chauhan, A. and Campoy, P. (2014). A supervised approach to electric tower detection and classification for power line inspection, Neural Networks (IJCNN), 2014 International Joint Conference on, IEEE, pp. 1970-1977.

Souza, Bruno A, C. E. G. (2016). Monitoramento e diagnóstico de isoladores poliméricos utilizando processamento digital de imagens de radiação infravermelha, Simpósio Brasileiro de Sistemas Elétricos . 